Supplement of Adv. Geosci., 45, 185-192, 2018

https://doi.org/10.5194/adgeo-45-185-2018-supplement

(c) Author(s) 2018. This work is distributed under

the Creative Commons Attribution 4.0 License.

(c) (1)

Supplement of

Conceptual model development using a generic Features, Events, and Processes (FEP) database for assessing the potential impact of hydraulic fracturing on groundwater aquifers

Alexandru Tatomir et al.

Correspondence to: Alexandru Tatomir (alexandru.tatomir@geo.uni-goettingen.de)

The copyright of individual parts of the supplement might differ from the CC BY 4.0 License. 
Tables

Table S1. The highest ranked features for the six focused scenarios.

\begin{tabular}{|c|c|c|c|c|c|c|}
\hline List of Features & S1 & S2 & S3 & S4 & S5 & S6 \\
\hline \multicolumn{7}{|l|}{ A. Features of the Natural System } \\
\hline \multicolumn{7}{|l|}{ 1. Hydrogeology } \\
\hline \multicolumn{7}{|l|}{ 1.1. Hydrocarbon bearing formation (Source) } \\
\hline Rock / Petrophysical properties of the hydrocarbon bearing formation & 4 & 4 & & & & \\
\hline Lithology & 4 & 4 & 4 & 4 & & 4 \\
\hline Intrinsic permeability & 4 & 4 & & 4 & 4 & \\
\hline Relative permeability & & 4 & 4 & 5 & 4 & \\
\hline Entry pressure & & 4 & 4 & 4 & & \\
\hline \multicolumn{7}{|l|}{ Stress and Mechanical properties } \\
\hline Heterogeneity of the hydrocarbon bearing formation & 4 & & & & & \\
\hline Fractures and faults within the hydrocarbon bearing formation & 4 & 4 & 4 & 5 & 4 & \\
\hline Porosity of the fracture & & & & 4 & & \\
\hline Intrinsic permeability of the fracture & 4 & 4 & & 4 & & \\
\hline Relative Permeability of the fractures & & 4 & & 4 & 4 & \\
\hline Fracture geometry & & 4 & & 4 & & \\
\hline \multicolumn{7}{|l|}{ 1.2. Fluids } \\
\hline Hydrocarbons & & & 4 & & & \\
\hline Natural formation water & & & 4 & & & \\
\hline Production fluids & & & 4 & & & \\
\hline Pore fluid composition within the fracking reservoir & & & 4 & & & \\
\hline Reservoir fluids & & & 4 & & & \\
\hline Other fluids & & & 4 & & & \\
\hline \multicolumn{7}{|l|}{ 1.3. Overburden } \\
\hline Porosity & & & & 4 & & \\
\hline Fractures and faults within the overburden & & 4 & & & & \\
\hline Relative Permeability of the fractures & & & & 4 & & \\
\hline Fracture geometry & & 4 & & 4 & & \\
\hline \multirow{2}{*}{\multicolumn{7}{|c|}{$\begin{array}{l}\text { Undetected features within the overburden } \\
\text { 2. Near surface environment (Receptors) }\end{array}$}} \\
\hline & & & & & & \\
\hline \multicolumn{2}{|l|}{ 2.1. Terrestrial environment } & & & & & \\
\hline Near-surface aquifers and surface water bodies & & & & & 4 & 4 \\
\hline \multicolumn{7}{|l|}{ B. Unconventional Hydrocarbon Extraction } \\
\hline \multicolumn{7}{|l|}{ 1. Hydro-fracturing fluid } \\
\hline 1.1. Hydraulic injection fluid properties & & & 4 & & & \\
\hline \multicolumn{7}{|l|}{ 1.2. Physical properties of injection fluid } \\
\hline Injection fluid additives & & & 4 & & & \\
\hline \multirow{2}{*}{\multicolumn{7}{|c|}{$\begin{array}{l}\text { 3. Site operation } \\
\text { 3.1. Drilling and completion }\end{array}$}} \\
\hline & & & & & & \\
\hline Horizontal wells & 4 & 4 & & & & \\
\hline \multicolumn{7}{|l|}{ 4. Site decommissioning } \\
\hline Abandoned wells & & & & 4 & & \\
\hline
\end{tabular}

Table S2. The highest ranked events for the six focused scenarios.

\begin{tabular}{|c|c|c|c|c|c|c|}
\hline List of Events & S1 & S2 & S3 & S4 & S5 & S6 \\
\hline \multicolumn{7}{|l|}{ 1. Operational Events } \\
\hline Hydraulic fracturing & 5 & 4 & 4 & 4 & & \\
\hline Out of zone / beyond pumping & & & & 4 & 4 & 4 \\
\hline Production & & 4 & 4 & 4 & & \\
\hline Seal failure & & 4 & & & 4 & \\
\hline \multicolumn{7}{|l|}{ 2. Natural events } \\
\hline Earthquakes & 4 & & & & & \\
\hline Cap rock failure & & 4 & & 4 & & \\
\hline 3. Accidents and unplanned events & & & & & & \\
\hline
\end{tabular}




\begin{tabular}{|c|c|c|c|c|c|}
\hline Overpressuring & 4 & 4 & 4 & 4 & \\
\hline Poor site characterization & & 4 & 4 & 4 & 4 \\
\hline Incorrect chemical mix released into fracking fluid & & & 4 & & \\
\hline Cementation poorly undertaken (spaces left) & & 4 & 4 & 4 & \\
\hline
\end{tabular}

Table S3. The highest ranked processes for the six focused scenarios.

\begin{tabular}{|c|c|c|c|c|c|c|}
\hline List of Processes & S1 & S2 & S3 & $\mathrm{S} 4$ & S5 & S6 \\
\hline \multicolumn{7}{|l|}{ 2. Hydraulics / Fluid Pressure Dominated } \\
\hline Fluid pressure exceeds rock fracking pressures generating new fractures & 4 & 4 & & 4 & & \\
\hline Fluid exceeds fault sealing pressures & 4 & 4 & & 4 & & \\
\hline Fluid pressure exceeds stability of part of the plant construction. & 4 & 4 & & & & \\
\hline Displacement of surrounding formation fluids & & 4 & & 4 & 4 & \\
\hline Buoyancy-driven flow & & 4 & & 4 & 4 & 4 \\
\hline Advection and co-migration of other gas & & & 4 & & & \\
\hline Water mediated transport & & & 4 & & 4 & \\
\hline Advection & & & 4 & 4 & 4 & 4 \\
\hline Dispersion & & & & 4 & 4 & 4 \\
\hline Diffusion & & & 4 & & & 4 \\
\hline Hydraulic and production fluids and the associated contaminants release processes & & & 4 & 4 & 4 & 4 \\
\hline \multicolumn{7}{|l|}{ 3. Chemical } \\
\hline Corrosive mixture attacks plant & & & 4 & & & \\
\hline Corrosive mixture attacks geology & & & 4 & & & \\
\hline Sorption and desorption & & & 4 & & & \\
\hline Mineral dissolution & & & 4 & 4 & & \\
\hline Heavy metal release & & & 4 & 4 & & \\
\hline \multicolumn{7}{|l|}{ 4. Mechanical } \\
\hline Soil and rock deformation around boreholes & 4 & 4 & & 4 & & \\
\hline Propagation of fractures beyond the target zone & 4 & 4 & 4 & 5 & & \\
\hline Fluid exceeds fault sealing pressures & 4 & 4 & & 5 & & \\
\hline Fault valving & 4 & 4 & & 4 & & \\
\hline Micro-cracking in the casing cements & & 4 & & 4 & & \\
\hline
\end{tabular}

Table S4. The key parameters and their variations for the 'regional fracturing fluid flow and stray gas migration' scenario.

\begin{tabular}{|l|l|l|}
\hline Parameters & Variation & Literature \\
\hline $\begin{array}{l}\text { Overburden thickness, } \\
m\end{array}$ & $1100-2100$ & (EIA 2011; 2011) \\
\hline Fault width, $m$ & $5-20$ & $\begin{array}{l}\text { (Gassiat et al. 2013; Kissinger et al. 2013b; Reagan et al. 2015; Pfunt } \\
\text { et al. 2016) }\end{array}$ \\
\hline $\begin{array}{l}\text { Overburden } \\
\text { permeability, } \mathrm{m}^{2}\end{array}$ & $\begin{array}{l}1 \times 10^{-18} \\
-1 \times 10^{-15}\end{array}$ & \begin{tabular}{l} 
(Gassiat et al. 2013) \\
\hline $\begin{array}{l}\text { Overburden anisotropy } \\
\text { ratio }\end{array}$
\end{tabular} $1-1000$ \\
\hline $\begin{array}{l}\text { Deep aquifer } \\
\text { permeability, } \mathrm{m}^{2}\end{array}$ & $\begin{array}{l}5 \times 10^{-16} \\
-5 \times 10^{-12}\end{array}$ & $\begin{array}{l}\text { (Nordbotten et al. 2005; Wigand et al. 2008; Birkholzer et al. 2009; } \\
\text { Michael et al. 2010; Vilarrasa et al. 2013) }\end{array}$ \\
\hline $\begin{array}{l}\text { Deep aquifer porosity } \\
0.05-0.35\end{array}$ & $\begin{array}{l}\text { (Nordbotten et al. 2005; Wigand et al. 2008; Birkholzer et al. 2009; } \\
\text { Michael et al. 2010; Vilarrasa et al. 2013) }\end{array}$ \\
\hline Fault permeability, $\mathrm{m}^{2}$ & $\begin{array}{l}2 \times 10^{-11} \\
-1 \times 10^{-10}\end{array}$ & $\begin{array}{l}\text { (Gassiat et al. 2013; Kissinger et al. 2013b; Birdsell et al. 2015; Pfunt } \\
\text { et al. 2016) }\end{array}$ \\
\hline Fault porosity & $0.08-0.12$ & (Myers 2012; Saiers and Barth 2012; Pfunt et al. 2016) \\
\hline Salinity, $\mathrm{kg} / \mathrm{m}^{3}$ & $150-350$ & (King 2012) \\
\hline
\end{tabular}




\section{References Supplementary Information}

Birdsell DT, Rajaram H, Dempsey D, Viswanathan HS (2015) Hydraulic fracturing fluidmigration in the subsurface: A review and expanded modeling results. Water Resour Res 51:7159-7188 . doi: 10.1002/2015WR017810.

Birkholzer J, Zhou Q, Tsang C (2009) Large-scale impact of CO2 storage in deep saline aquifers: A sensitivity study on pressure response in stratified systems. Int J Greenh Gas Control 3:181-194 . doi: 10.1016/j.jjggc.2008.08.002

EIA U (2011) Review of emerging resources: US Shale gas and shale oil plays. Energy Inf Adm US Dep Of

Freeze R, Cherry J (1979) Groundwater, 604 pp

Gassiat C, Gleeson T, Lefebvre R, McKenzie J (2013) Hydraulic fracturing in faulted sedimentary basins: Numerical simulation of potential contamination of shallow aquifers over long time scales. Water Resour Res 49:8310-8327 . doi: 10.1002/2013WR014287

King G (2012) Hydraulic fracturing 101: what every representative, environmentalist, regulator, reporter, investor, university researcher, neighbor and engineer should know about. SPE Hydraul Fract Technol Conf

Kissinger A, Helmig R, Ebigbo A, Class H, Lange T, Sauter M, Heitfeld M, Klünker J, Jahnke W (2013) Hydraulic fracturing in unconventional gas reservoirs: Risks in the geological system, part 2: Modelling the transport of fracturing fluids, brine and methane. Environ Earth Sci 70:3855-3873 . doi: 10.1007/s12665-013-2578-6

Michael K, Golab A, Shulakova V, Ennis-King J, Allinson G, Sharma S, Aiken T (2010) Geological storage of $\mathrm{CO} 2$ in saline aquifers $-\mathrm{A}$ review of the experience from existing storage operations. Int J Greenh Gas Control 4:659-667 . doi: 10.1016/j.ijggc.2009.12.011

Myers T (2012) Potential Contaminant Pathways from Hydraulically Fractured Shale to Aquifers. Groundwater 50:872-882

Neuzil C (1994) How permeable are clays and shales? Water Resour Res 30:145-150

Nordbotten JM, Celia MA, Bachu S (2005) Injection and Storage of CO 2 in Deep Saline Aquifers: Analytical Solution for CO 2 Plume Evolution During Injection. Transp Porous Media 58:339-360 . doi: $10.1007 / \mathrm{s} 11242-004-0670-9$

Pfunt $\mathrm{H}$, Houben G, Himmelsbach $\mathrm{T}$ (2016) Numerical modeling of fracking fluid migration through fault zones and fractures in the North German Basin. Hydrogeol J 24:1343

Reagan MT, Moridis GJ, Keen ND, Johnson JN (2015) Numerical simulation of the environmental impact of hydraulic fracturing of tight/shale gas reservoirs on near-surface groundwater: Background, base cases, shallow reservoirs, short-term gas, and water transport. 1-31

Saiers JE, Barth E (2012) Comment on "Potential Contaminant Pathways from Hydraulically Fractured Shale to Aquifers" by T. Myers. In: Ground Water 
Vilarrasa V, Silva O, Carrera J, Olivella S (2013) Liquid CO2 injection for geological storage in deep saline aquifers. Int J Greenh Gas Control 14:84-96 . doi: 10.1016/j.ijggc.2013.01.015

Wigand M, Carey JW, Schütt H, Spangenberg E, Erzinger J (2008) Geochemical effects of CO2 sequestration in sandstones under simulated in situ conditions of deep saline aquifers. Appl Geochem 23:2735-2745 . doi: 10.1016/J.APGEOCHEM.2008.06.006

(2011) United States. Energy Information Administration, and Vello Kuuskraa. World shale gas resources: an initial assessment of 14 regions outside the United States. US Department of Energy 